\title{
Constructing Home Monitoring System with Node-RED
}

\author{
Chien-Yu Lu, ${ }^{1}$ Fei-Hsu Chen, ${ }^{1}$ Wen-Chiung Hsu, ${ }^{1}$ Yu-Qiang Yang, ${ }^{1}$ and Te-Jen $\mathrm{Su}^{2,3 *}$ \\ ${ }^{1}$ Department of Industrial Education and Technology, National Changhua University of Education, \\ Changhua City 500, Taiwan, ROC \\ ${ }^{2}$ Department of Electronic Engineering, National Kaoshiung University of Science and Technology, \\ Kaohsiung City 80778, Taiwan, ROC \\ ${ }^{3}$ Ph.D Program in Biomedical Engineering, Kaohsiung Medical University, Kaohsiung City 807, Taiwan, ROC
}

(Received September 25, 2019; accepted February 19, 2020)

Keywords: home monitoring system, Node-RED, MQTT, database, remote monitoring

The aim of this study is to design a home monitoring system with a simple and clear visual interface that integrates home appliances, disaster prevention, and surveillance facilities. Furthermore, the Node-RED visual development environment was utilized to create the system, and the users will be able to configure the environmental parameters by themselves with its simple and graphical programming interface, to customize the user interface. In this study, we utilize the Node-RED development software installed on a personal computer and the Message Queuing Telemetry Transport (MQTT) to communicate and exchange data with home devices under the publish/subscribe mode, so that the stability of data transmissions can be ensured while more resources are saved; in addition, MySQL database management tools are used to access the data stored in the home devices for the statistics and analysis of changes in environmental data. Moreover, the HyperText Transfer Protocol (HTTP) is also used to acquire various information from the government's open source data platform, and the information will be integrated and displayed on the Node-RED webpage interface. Since Node-RED uses a webpage interface, the devices that can use web browsers to view webpages, such as computers and smart phones, can thus be used to remotely monitor the Node-RED program. Finally, we utilize the Node-RED development software installed on a personal computer and MQTT to communicate and exchange data with the home devices under the publish/subscribe mode. The smart home system is divided into input and output parts, which control the electrical appliances and read the sensor information. Ten switches on the Web control interface are used to control the living room, kitchen, balcony, and bedroom light switches, air-conditioning, heating, exhaust fans, outdoor lights, doors, and alarm switches.

\section{Introduction}

With the popularity of internet devices and smart devices nowadays, the transmission speed and amount of information are already beyond our imagination. In particular, the rapidly advancing wireless communication technology has enabled us to access the internet without

*Corresponding author: e-mail: sutj@nkust.edu.tw

https://doi.org/10.18494/SAM.2020.2686 
the limitation of location, as we can enjoy the convenience of accessing wireless internet from offices, homes, cars, hotels, and cafes. The economic model in the industry has been gradually stepping into the era of intelligence-oriented from computational- and communication-oriented. The Internet of Things will be the key for the future as the industry will combine with artificial intelligence (AI) technology and connect to terminal devices through data transmission on the internet to carry out comprehensive intelligent application services. ${ }^{(1)}$

Over the past few years, smart home application services have been continuously developing, and the number of products and smart home devices on the market has increased. Ovum's "Smart Home Devices Forecast: 2016-21" indicated that smart home devices would be used in almost 90 million homes worldwide in 2016. On the basis of Ovum's forecast report, the key factor of the product penetration rate for smart home devices is the availability of internet broadband, which is also the reason why Wi-Fi has become the most popular home wireless technology around the world. ${ }^{(2)}$

Smart home applications can be seen everywhere; however, the most important task is to create devices that can fulfill a user's needs and use habits in the home environment. These home devices can automatically connect to the internet, automatically detect and respond to changes in the environment, as well as collect environmental information via the sensing components and connect to the system; the data will be implemented in the algorithm and AI program to analyze the implementation results to create a scenario application, such as the configuration of temperature and light, and the service of turning on and off the air-conditioner, heater, and lighting equipment, which make these home devices more intelligent and userfriendly. ${ }^{(3)}$

On the basis of this, a smart home application service was created using Node-RED with the implementation of environmental sensing data, so that users can remotely control the surrounding devices via their smartphones or other internet-connected devices in the most simple and intuitive way, as well as to automatically adjust the home devices. ${ }^{(4)}$

\section{System Structure}

In this section, we describe the software used and the function of the smart home service, including Node-RED program coding, data transmission, cloud visual recognition, and database. The core of this study is Node-RED, which was developed by IBM's Emerging Technology Services team using visual IoT development tools. Node.js. is an open source and cross-platform environment for JavaScript, which makes the JavaScript applicable to programming at the server end. ${ }^{(5)}$ The Node-RED visual development environment is based on the flow, and users can write the program through a combination of various nodes. These nodes can be hardware equipment, web application programming interfaces (Web APIs) or cloud services, and the coding environment is shown in Figs. 1(a) and 1(b). The browser is the development interface of Node-RED. The flow can be edited in the middle of the screen using the node on the left panel, and the information of all the nodes can be viewed on the right panel. The tool of the graphical dashboard can also be used to control devices and monitor the information of various devices within the same domain. 


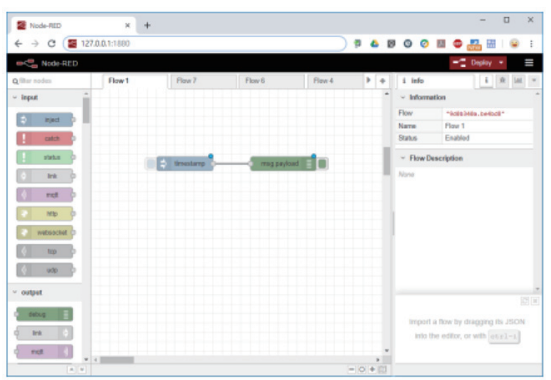

(a)

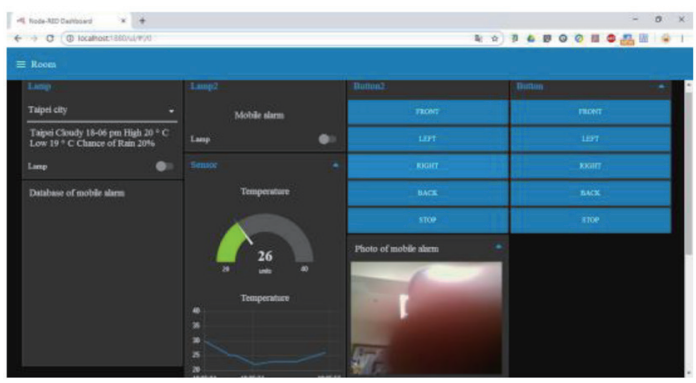

(b)

Fig. 1. (Color online) (a) Node-RED environment and (b) Node-RED board.

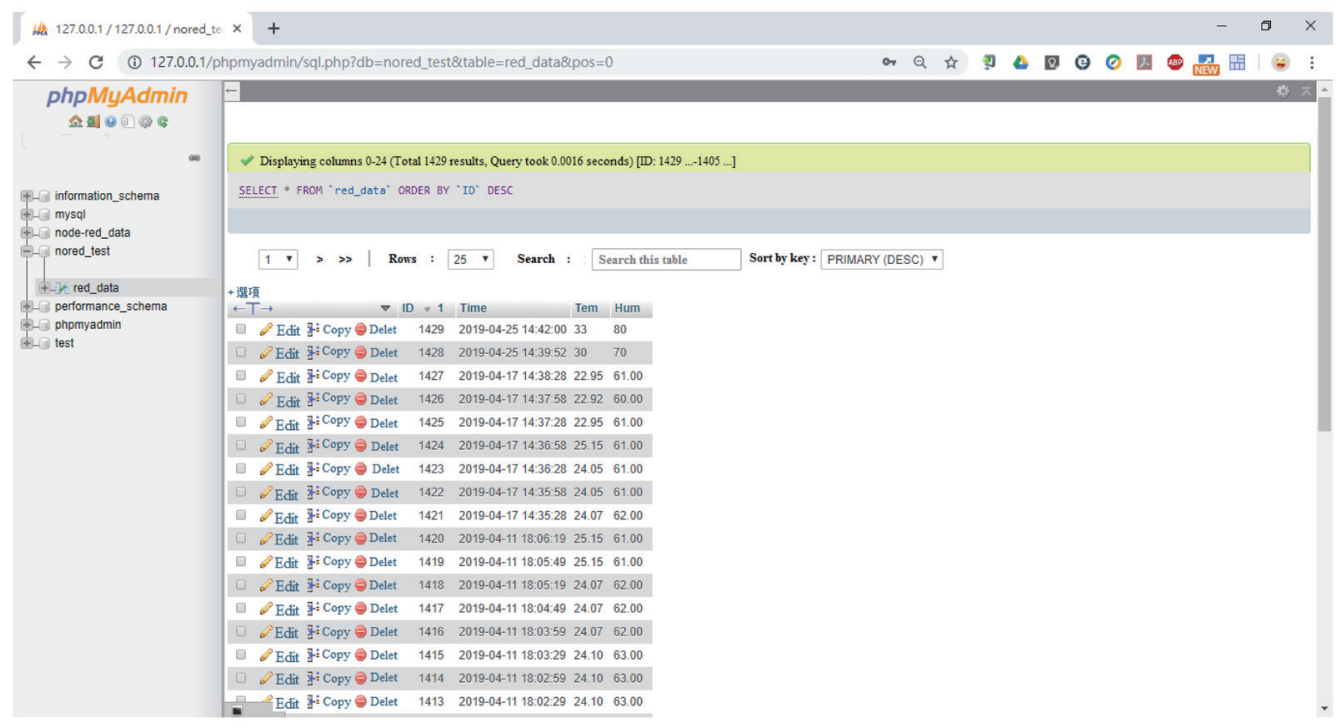

Fig. 2. (Color online) Management interface of phpMyAdmin.

For data transmission, Mosquitto is used to create the Message Queuing Telemetry Transport (MQTT) Broker and provides one-to-many message delivery through the publish/ subscribe mechanism, so that the information can be shared and exchanged amongst the smart home devices. ${ }^{(6)}$ In addition, the HyperText Transfer Protocol (HTTP) is used to acquire the real-time monitoring data on weather and air quality from the government's open source data platform, ${ }^{(7,8)}$ and the information will be displayed on the Node-RED dashboard after compilation.

The IBM Watson visual recognition service is used for the cloud image and video recognition, the face image is analyzed using a deep learning algorithm, ${ }^{(9)}$ and the snapshot of alarms can be quickly published on the webpages and LINE account or groups to obtain the identification result via Node-RED. The database uses phpMyAdmin, which is a Web-Based MySQL database management tool available on the web server, and enables the users to manage the MySQL database by using the webpage interface shown in Fig. $2^{(10)}$ and access information via Node-RED. 


\section{Monitoring System}

In this section, we introduce the hardware architecture of the system and the programming architecture. The first introduces the hardware architecture of the server and home devices. Also, we introduce the external connection facility and sensors of the home monitoring system. Finally, we introduce the programming architecture of the home monitoring system, which integrates home appliances, disaster prevention, and surveillance facility with Node-RED, as shown in Ref. 11.

After using the Node-RED software at the server end to create a Web control interface and program and publish/subscribe topics and information through MQTT Broker to remotely (Wi-Fi) control the switch of the relay with the Web control interface and read the data of the sensor. In addition, the alarm system is connected to the webcam software through Node-RED with human body and disaster prevention sensors, and to the database software and accesses various sensor data for the control actions; moreover, this information will be classified and displayed on the webpage and the alert messages will be published on the individual's LINE account or groups.

The home monitoring system is as shown in the top view of a house model in Fig. 3. It includes four main sections, namely, the living room, balcony, kitchen, and bedroom, and each section is equipped with external connection equipment and sensors with the NodeMCU demoboard. The living room has the temperature and humidity sensor, fire sensor, electric door, and magnetic switch installed; the kitchen has the gas sensor and exhaust fan installed; the balcony has the light and human body sensor installed; and the bedroom has the relay switch installed.

The main hardware architecture of the home monitoring system is the server, the Node-RED software installed on a notebook computer, and the home monitoring server. Furthermore, the

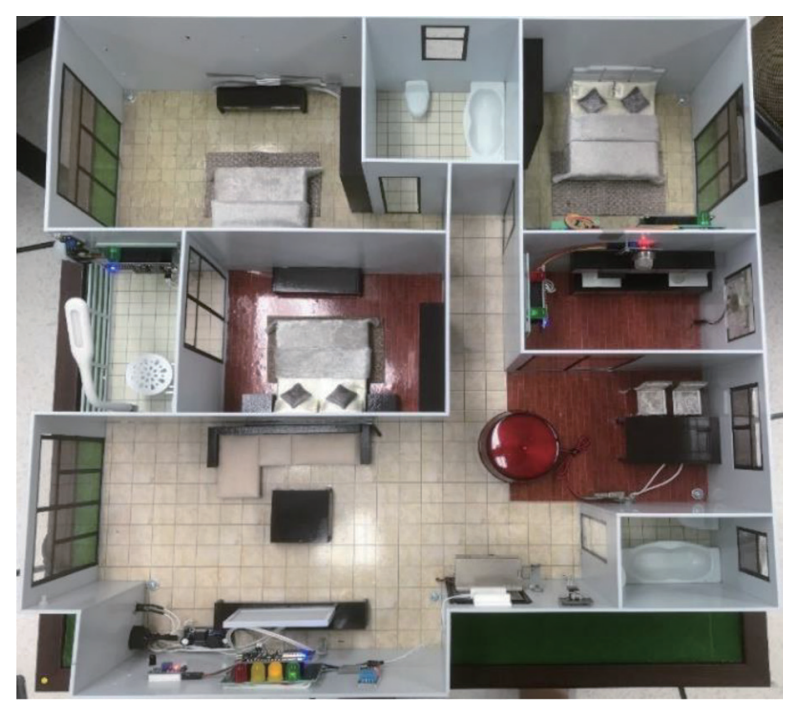

Fig. 3. (Color online) Top view of a house model. 
wireless router and the core of the home appliance control NodeMCU demoboard are used for wireless data transmission via Wi-Fi. In this research, we utilize the NodeMCU demoboard as the home appliance controller of this system. The system connects to the NodeMCU and uses it as a controller through the Wi-Fi wireless transmission; with the embedded system, the smart home system can control the home appliances with the capability of its computational program. The NodeMCU is based on the demoboard of Espressif Wi-Fi chip ESP8266 ESP12, which uses Lua Script for program coding. It is an open source hardware and an interactive, low-cost, and intelligent demoboard with a Wi-Fi module. Its demoboard is equipped with a CH341 chip and a USB socket, which is plug-and-play, as shown in the diagram. Its hardware specifications and pins are shown in Table 1 and Fig. 4 below.

Table 1

NodeMCU hardware specifications.

\begin{tabular}{|c|c|}
\hline \multicolumn{2}{|c|}{ Supports wireless $802.11 \mathrm{~b} / \mathrm{g} / \mathrm{n}$ standards } \\
\hline \multicolumn{2}{|c|}{ Supports three operating modes STA/AP/STA+AP } \\
\hline \multicolumn{2}{|c|}{ Built-in TCP/IP protocol, support for multiple TCP Client connections (5 MAX) } \\
\hline \multicolumn{2}{|c|}{ Supports UART/GPIO data communication port } \\
\hline Communication port voltage & $3.3 \mathrm{~V}$ \\
\hline D0-D8, SD1-SD3 & Used for GPIO, PWM, IIC, etc., with $15 \mathrm{~mA}$ port drive capability \\
\hline AD0 & 1 ADC channel \\
\hline Power input & 4.5-9 V (10 V MAX); support USB power supply \\
\hline Operating current & In operation: $\approx 70 \mathrm{~mA}(200 \mathrm{~mA} \mathrm{MAX}) ;$ standby: $<200 \mu \mathrm{A}$ \\
\hline Transmission rate & $110-460800$ bps \\
\hline Operating temperature & $-40-125^{\circ} \mathrm{C}$ \\
\hline Flash size & $4 \mathrm{MB}$ \\
\hline
\end{tabular}

\section{PIN DEFINITION}

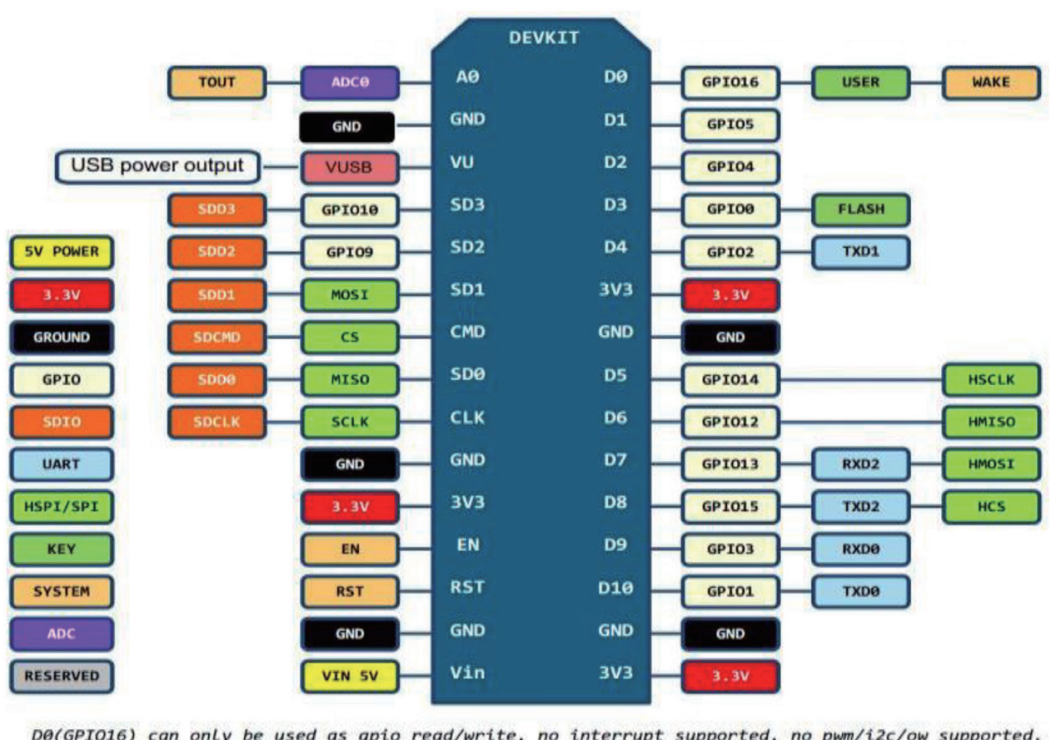

Fig. 4. (Color online) Definition of NodeMCU pins. 
In addition to the main hardware such as the server and NodeMCU demoboard, this study also requires some external connection equipment and sensors, including a webcam, relay module, temperature and humidity sensor, light sensor, flame sensor, gas sensors, and infrared human-body sensor. First, the webcam is used as the source of video input for the home monitoring system, and the system is connected to the server via USB, so that the remote user can monitor the surrounding home environment at any time. Furthermore, the program with displacement alarms and the image recognition system are also utilized to identify the moving objects in the surrounding area.

The main program functions and program architecture of the home monitoring system use Node-RED to establish a home monitoring system, and the operating mode of the program can be quickly understood through the process flow chart. This system includes six subsystems; the home appliance control system, sensor monitoring, image recognition system, open data reading system, database system, and LINE notification system. We can see the functional architecture diagram of the system from the above diagram, as well as the process flow chart of the home monitoring system, as shown in Fig. 5.

\section{Experimental Results}

The web control interface of this system is shown in Fig. 6. It is divided into several blocks, which read data and acquire open data for the sensors, to control the home appliances. In this

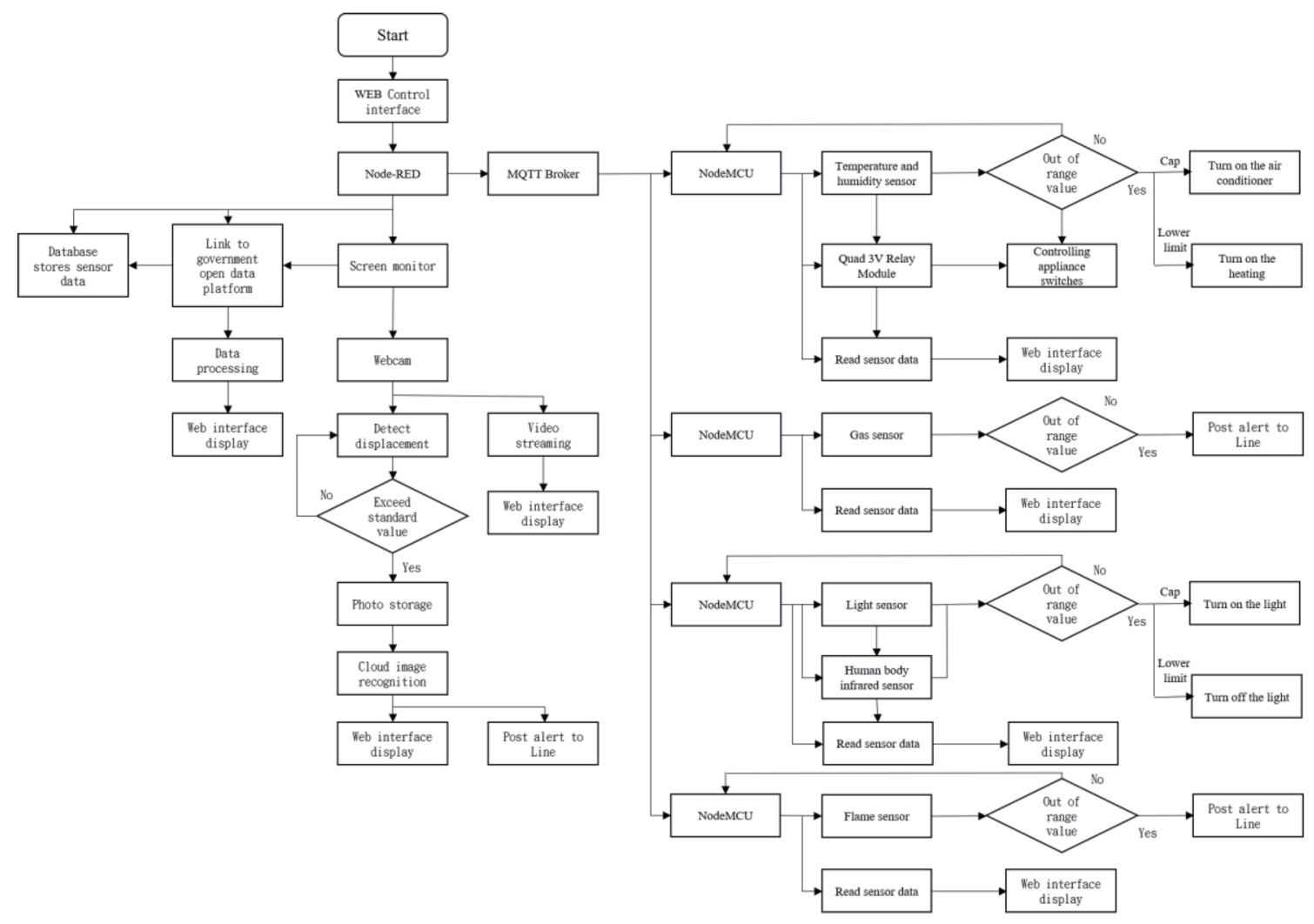

Fig. 5. Process flow chart of the home monitoring system. 


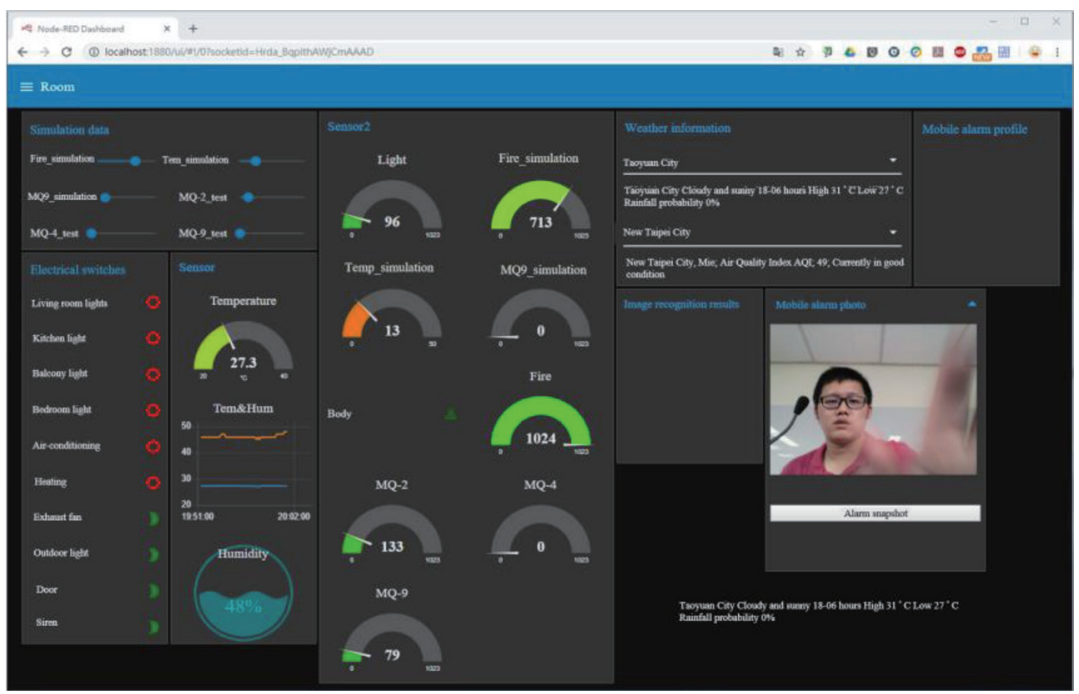

Fig. 6. (Color online) Web control interface.

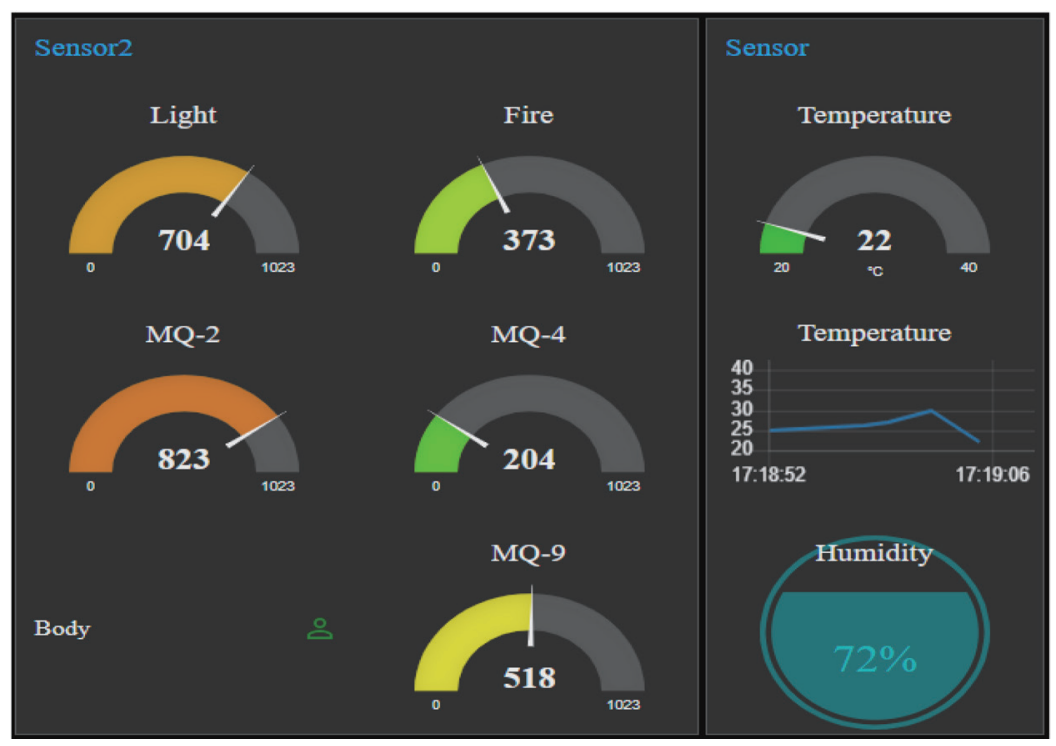

Fig. 7. (Color online) Sensor meters of web control interface.

section, we describe in detail the integration and implementation of the home appliance control system, sensor system, open data reading system, database system, and image recognition system.

First, the smart home system is divided into input and output parts, which control the electrical appliances and read the sensor information. Ten switches on the Web control interface are used to control the living room, kitchen, balcony, and bedroom light switches, and air-conditioning, heating, exhaust fans, outdoor lights, doors, and alarm switches.

Next, the sensor block is divided into temperature, humidity, illumination, flame, human body, and three types of gas sensing, which are presented in the form of meters on the web control interface. As shown in Fig. 7, the current sensor value can be obtained in real time. 
Also, an open data reading system is connected to the meteorological data open platform of the Central Meteorological Bureau of the Ministry of Communications and the environmental resource data open platform of the Environmental Protection Agency of the Executive Yuan to obtain weather forecast information and air quality information. The drop-down menu at the top of the web control interface allows one to view the weather information of the counties and cities, and below is the air quality indicator and the current air pollution status.

Then, the database system organizes the obtained sensor data and stores it in the database management system, so that one can view the data and detect abnormal conditions in the future. The MQTT node is used to connect the function nodes, to obtain various sensor data, to organize and merge them, and finally, to link and store them in the database system. Finally, the image recognition system is divided into three blocks on the Web control interface: the mobile alarm photos, mobile alarm data, and image recognition results. First use the camera program's image streaming and displacement camera function to display the streaming image on the web control interface. Then, detect if there are new files in the displacement alarm photo folder, and arrange the results to be displayed in the web control interface. Finally, the alarm photos are uploaded to the cloud image recognition service, and the results are displayed on the web control interface and published on the individual's LINE account or groups.

\section{Conclusions}

In this study, we integrated the home appliance control, sensors, data access, and architecture of image recognition, and utilized the Node-RED and NodeMCU software and hardware devices to establish an IoT system. This system can monitor the temperature, humidity, light, flame, and gas conditions in the surrounding area, and it can remotely and automatically control the home appliances and equipment; moreover, it also combines with image recognition, motion detection, and LINE notification systems. The Node-RED program is structured at the server and uses the wireless network and MQTT communication protocol to acquire the sensing data in the environment and transmit the control signals for the home appliances, as well as store various data in the database. The user can access the web control interface on the remote network devices to instantly monitor the home environment and receive any disaster prevention alarm via LINE notification, so as to complete the study of the comprehensive home monitoring system. The home monitoring system developed in this study still has much room for improvement. A home access control system is expected to be added in the future and combined with the functions of image recognition and alarm, so that the system can automatically determine whether the person at the door is the owner of the house, whereby it opens the door, and sends the personnel access information to LINE. In addition, we also hope to enhance the security of the Node-RED system. We will first increase the number of user's management permissions and thoroughly conduct the user's identity authentication and access control, and encrypt the transmission data of sensors to prevent data breaches. Lastly, we expect the system to be combined with the home medical care system and AI system to collect and analyze the user's physiological data, and then implement the algorithm analysis to evaluate the user's physical condition for future illness prevention. 


\section{References}

1 W. C. Tsai and H. C. Yu: Master's Thesis, Research on the Trend of Internet of Things and Government's Policies, Institute of Science and Technology Management 2011: https://lib.nctu.edu.tw

2 Smart Home Devices Forecast 2017: https://ovum.informa.com/resources/product-content/smart-homedevices-forecast-201621 (accessed June 2019).

3 D. M. Han and J. H. Lim: IEEE Trans. Consum. Electron. 56 (2010) 1403. https://ieeexplore.ieee.org/ document $/ 5606276$

4 Node-RED: OpenJS Foundation. https://nodered.org

5 Node.js Foundation: https://nodejs.org/en/ (accessed June 2019).

6 R. Lu and Y. Liao: Internet of Things: Node-RED Internet of Things visualization (Wunan, Taipei, Taiwan, 2017).

7 Eclipse Mosquitto: https://mosquitto.org/ (accessed June 2019).

8 Central Weather Bureau, MOTC: https://opendata.cwb.gov.tw/ (accessed June 2019).

9 Environmental Protection Administration, Executive Yuan Environmental Protection Administration, Executive Yuan: https://opendata.epa.gov.tw/Data/Contents/AQI/ (accessed June 2019).

10 Watson Visual Recognition (IBM Watson): https://www.ibm.com/watson/services/visual-recognition/ (accessed June 2019).

11. phpMyAdmin: https://www.phpmyadmin.net/ (accessed June 2019).

\section{About the Authors}

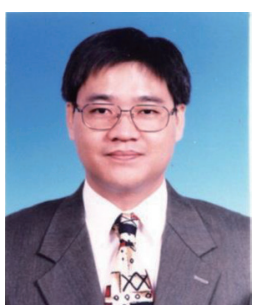

Chien-Yu Lu received his B. S. degree from the National Changhua University of Education, Taiwan, and M. S. degree from the National Cheng Kung University, Taiwan, in 1992 and 1995, respectively, and Ph. D. degree in the Department of Electrical Engineering, National Cheng Kung University, Taiwan, R.O.C, in 2004. He has been with the National Changhua University of Education, Changhua, Taiwan, R.O.C., since 2011, where he is now a professor. His current research interests include nonlinear systems, neural networks, stability theory and IoT.

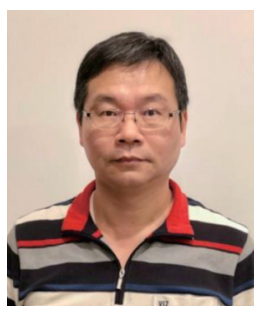

Fei-Hsu Chen received his B. S. and M. S. degrees from the National Changhua University of Education, Taiwan R.O.C, in 1992 and 1994. After graduation, he worked at the Industrial Technology Research Institute from 1994 to 2007. Since 2007, he has been working for the Foxconn technology group and is responsible for leading the R \& D team in PC base motion control, CNC system and servo drive control development. (st168chen@gmail.com)

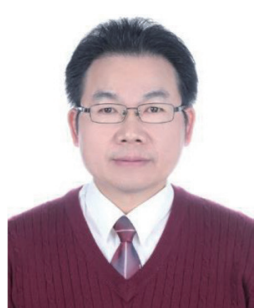

Wen-Chiung Hsu received his B. S. and M. S. degrees from the National Changhua University of Education, Taiwan R.O.C, in 1992 and 1994. After graduation, he worked at the National Erh Lin and Commercial Vocational High School from 1994 to 1997. Since 1997, he has been working for the Taichung Municipal Feng Yuang Commercial High School and is currently the practical director responsible for technical education. His current research interest is IoT. 


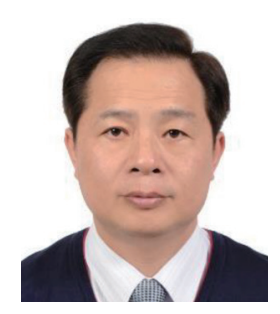

Yu-Qiang Yang received his B. S. degree from the National Chin Yi University of Technology, Taiwan, and M. S. degree from the National Changhua University of Education, Taiwan, respectively, and $\mathrm{Ph}$. D. degree in the Department of Electrical Engineering, National Changhua University of Education, Taiwan, R.O.C, in 2015. He has been with the National Changhua University of Education, Changhua, Taiwan, R.O.C., since 2015, where he is now an assistant professor. He is also general manager of Feilo Power Electronics Co., Ltd. His current research interests include automation integration and IoT.

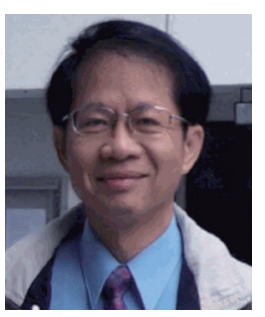

Te Jen Su received his Ph.D. degree in electrical engineering from National Cheng-Kung University Tainan, Taiwan, in 1989. He is currently a professor with the Department of Electronic Engineering, National Kaohsiung University of Sciences and Technology, Kaohsiung City, Taiwan, R.O.C. and Kaohsiung Medical University, Kaohsiung City, Taiwan, R.O.C. His research interests include intelligent control systems, embedded processor design and satellite communication systems. 